\title{
MISCELÁNEA
}

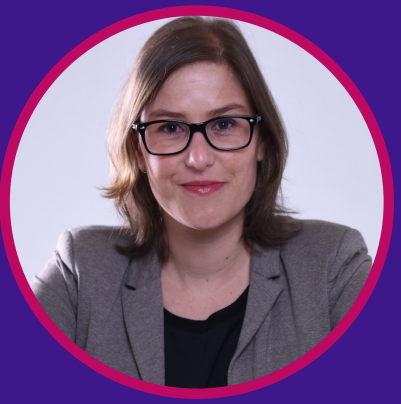

\section{Puesta en marcha de un nuevo Grado online en Publicidad en el contexto de pandemia sanitaria}

\author{
Launching a new online Advertising degree in the context of the \\ Covid-19 pandemic
}

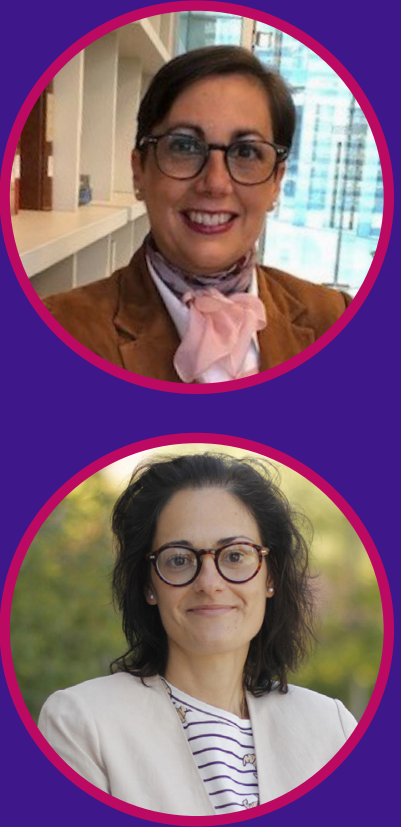

Dra. Erika Fernández Gómez

Universidad Internacional de la Rioja

(España)

erika.fernandez@unir.net

ORCID: https://orcid.org/0000-0002-7088-1814

Dra. Beatriz Feijoo Fernández

Universidad Internacional de la Rioja

(España)

beatriz.feijoo@unir.net

ORCID: https://orcid.org/0000-0001-5287-3813

Recibido: $\mathbf{3 0}$ de marzo de 2021.

Aceptado: 15 de abril de 2021.

Publicado: 31 de mayo de 2021.

\section{Dra. Adela López Martínez}

Universidad Internacional de la Rioja

(España)

adela.lopez@unir.net

ORCID: https://orcid: 0000-0003-3428-4868

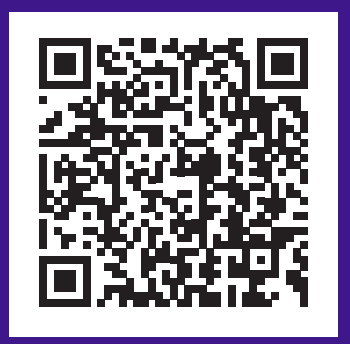

Esta obra está bajo una licencia internacional Creative Commons Atribución-NoComercial-SinDerivadas 4.0.

\section{DOI: https://doi.org/10.21555/rpc.v0i1.2364}

Cómo citar: Fernández Gómez, E. ., López Martínez, A. ., \& Feijoó Fernández, B. . (2021). Puesta en marcha de un nuevo Grado online en Publicidad en el contexto de pandemia sanitaria. RPC, (1), 212-222. https://doi.org/10.21555/rpc. voi1.2364

Revista Panamericana de Comunicación, Año 3, N. 1, enero-julio 2021, pp. 212-222

\section{Videopresentación}




\section{RESUMEN}

En España, en el curso 2019-2020 existían 35 titulaciones de Publicidad y Relaciones Públicas, de ellas, solo tres ofrecían la posibilidad de cursarse online, una modalidad que facilita la personalización educativa, que cada estudiante se desarrolle en función de su capacidad, sus intereses, sus aficiones, su ritmo de aprendizaje, sus circunstancias familiares y sociales, algo vital en un contexto de pandemia sanitaria. Este estudio comparte la experiencia de implantar un nuevo Grado online en Publicidad marcado por el Covid-19, así como las buenas prácticas puestas en marcha en el inicio del primer curso académico (2020-2021), influido por esta situación excepcional. La metodología online empleada cuenta ya con el aval de una universidad con diez años de experiencia virtual, pero se hacía frente a una nueva situación marcada por el contexto de la pandemia sanitaria y que supuso, entre otros factores, incorporar a un perfil de alumno más joven. Se muestra cómo se ha respondido a la consecución de competencias clave para este perfil de estudio como son las habilidades de comunicación oral, el trabajo en equipo y la capacidad de conceptualización bajo esta circunstancia sanitaria tanto en el diseño de los contenidos, como en la preparación de las sesiones en directo, entre otras dinámicas pedagógicas, para conseguir un índice de satisfacción entre el alumnado de 8,7/10.

Palabras clave: Grado, Publicidad, docencia virtual, pandemia, Covid-19; competencias

\section{A BSTRACT}

In Spain, there were 35 degrees in Advertising and Public Relations in the 2019-2020 academic year, with only three of them being taught online. Online learning facilitates educational personalization, as students progress according to their ability, interests, hobbies, learning pace, family and social circumstances, something that proved to be vital in the context of a health pandemic. This study shares the experience of starting a new online degree in Advertising in times of Covid-19, as well as the best practices implemented in its first academic year (2020-2021), in such an exceptional context. Whereas the university has been providing online instruction for ten years, the pandemic made it face new circumstances, such as a younger student profile. This paper describes how the key competences of this first year of study were addressed: developing oral communication skills, teamwork and the ability to conceptualize. It focuses both in the design of the contents and in the preparation of the live sessions in a pandemic context. At the end of this first term, the degree achieved a student satisfaction score of 8.67/10.

Keywords: degree, Advertising, virtual teaching, pandemic, Covid-19; competences. 


\section{LA INSTITUCIÓN UNIVERSITARIA}

【 ( Universidad" dice relación con universalidad, con el saber reconocido por todos como tal, con aquello que está en el origen de la cultura misma y que todo hombre aspira a conocer. En su sentido clásico, la Universidad solo tiene lugar cuando se constituye una "comunidad" de diálogo entre aquellos que, abiertos a toda verdad, se dedican al saber superior.

La universidad es algo más que el lugar donde se aprende un trabajo concreto: es donde los jóvenes se plantean las grandes preguntas y buscan respuestas, donde la imaginación y el amor por la sabiduría se encuentran con la ciencia, donde se hace avanzar el conocimiento y donde la inteligencia humana se abre a la universalidad del saber.

Es misión de la universidad formar personas íntegras. Si bien las universidades difieren con respecto a lo que significa la integridad, lo cierto es que todas aspiran -de una u otra manera- a que los individuos que egresan de sus aulas sean mucho más que un cúmulo de conocimientos dispersos. Todas quieren formar personas con habilidades para adaptarse a un mundo laboral cambiante y, sobre todo, con la rectitud moral para tomar las decisiones correctas ante estos cambios (López y Nagel, 2018).

Las universidades del mundo vienen, desde hace varios años, cuestionando cómo forman a sus alumnos. El proceso puntal de este cuestionamiento se dio en Europa, a principios del tercer milenio, con el llamado "Proceso Bolonia", que impulsó a las universidades europeas a formular su educación en términos de las "competencias" que debían tener sus egresados luego de pasar por su proceso de aprendizaje. Este proceso ha tenido una gran influencia -para bien o para malsobre las universidades en América Latina.

\section{LA UNIVERSIDAD ON LINE}

Desde hace unos años la universidad se ha planteado el cambio, no solo en su formato y estructura clásicos, sino en su propio enfoque de la educación. En realidad, es una necesidad subyacente a los cambios de la sociedad, mayormente mediatizados por el impacto y la emergencia del uso de las Tecnologías de la Información y la Comunicación en los distintos ámbitos de la vida cotidiana. En este contexto surgen las universidades virtuales.

Todavía es difícil dar una buena definición de lo que es una universidad virtual porque hay muchas instituciones de educación superior que están probando diferentes modalidades de formación en donde se utilizan diferentes grados de virtualización de la organización.

El término "universidad virtual" debería englobar un concepto sistémico de la universidad ofrecida a los estudiantes y la comunidad docente e investigadora, un concepto integral de universidad en sí misma, suficientemente distinto del término "campus virtual" (Sangrá, 2001). Este autor distingue entre a) Universidad presencial que ofrece elementos de virtualidad; b) Universidad presencial con extensión universitaria virtual; c) Espacios compartidos de cursos virtuales que ofrecen las universidades presenciales; d) Universidad virtual adosada a la universidad tradicional y e) Universidad virtual como organización virtual.

Al centrarse en este último concepto, surge la pregunta por las razones que justifiquen la existencia de la universidad virtual. Y encontramos múltiples: a) la necesidad de flexibilizar el rígido esquema curricular clásico para hacer innovación educativa; b) el deseo de llegar a un número cada vez mayor de personas y atender así nuevas demandas; c) la posibilidad de satisfacer todas las necesidades educativas de la población; d) la globalización y la liberalización de la economía; e) los nuevos canales existentes para que la universidad ejerza su función social... (Moreno-Castañeda et al., 2010).

La propuesta de la universidad on line facilita la personalización educativa, coherente con el hecho de que cada individuo es único, irrepetible y autónomo. Hace posible que cada estudiante se desarrolle en función de su capacidad, sus intereses, sus aficiones, su ritmo de aprendizaje, sus circunstancias familiares y sociales... Así es más fácil el trabajo independiente, la reflexión personal, el estudio individualizado, la investigación, el ejercicio de la creatividad, el desarrollo del sentido crítico. 
Además, teniendo en cuenta la dimensión social del hombre y, por tanto, el hecho de que la educación se basa en la relación entre personas, la universidad on line también puede contemplar el trabajo colaborativo, que desarrolla el sentimiento de "nosotros", fortaleciendo el espíritu de grupo y atenuando el egoísmo; reemplaza la competitividad por la cooperación; ayuda a vencer temores, tensiones e inhibiciones; enseña a ver los temas desde otros puntos de vista y a escuchar de modo compresivo propiciando el diálogo; facilita la adquisición de actitudes de respeto y tolerancia hacia los demás; y estimula el intercambio de ideas, promoviendo el desarrollo de la iniciativa y la creatividad personal (Castellanos-Sánchez y Martínez de la Muela, 2013).

En la universidad online se evidencia aún más la idea clásica de que el profesor es un facilitador (recordemos la comparación socrática de la tarea del educador con la de una matrona). Lo importante es el trabajo y el esfuerzo del estudiante, su proceso de aprendizaje. La enseñanza virtual pone en evidencia la necesidad de resaltar el desarrollo de las competencias: no basta saber, hay que saber hacer. En consecuencia, crea espacios propicios para enseñar a reflexionar, pensar críticamente, buscar información para resolver un problema: el modelo educativo está centrado en el aprendizaje del estudiante, no en la enseñanza del profesor (Tourón y Martín Rodríguez, 2019).

La experiencia docente que se comparte en este texto se desarrolla al amparo de una universidad virtual de carácter privado con sede en España, que se dedica a la impartición de estudios superiores (grados y postgrados de carácter oficial) bajo la modalidad on line y que cuenta con un campus virtual de desarrollo propio.

Desde su nacimiento en 2009, esta universidad ha basado su actividad académica en un modelo educativo centrado en la educación personalizada y en los avances tecnológicos de la sociedad digital. Así, ofrece clases, seminarios y tutorías en directo virtual, de tal modo que los profesores y los alumnos, mediante una cámara y un micrófono, a través del ordenador, pueden interactuar de manera sincrónica, rompiendo con la idea de impersonalidad y frialdad asociada generalmente con la educación a distancia. Dicho de otro modo, se trata de un modelo educativo cuyo fundamento es la persona: tiene en cuenta su naturaleza, sus necesidades, sus intereses y su meta no es otra que la plenitud personal (Castellanos-Sánchez y Martínez de la Muela, 2013). Se combina la tecnología (la posibilidad de asistir a clases on line desde cualquier lugar con un horario flexible) con la relación personal entre profesor y alumno y de este último con un tutor que lo acompaña y orienta en todo su proceso formativo.

\section{UN NUEVO GRADO ON LINE EN PUBLICIDAD}

La tarea que se ha venido desempeñando en las universidades con el título de Graduado en Publicidad y Relaciones Públicas sigue siendo fundamental para poder hacer frente a los cambios que las nuevas tecnologías han supuesto en la relación de las marcas y los consumidores.

Nos encontramos en un mercado altamente competitivo donde cada vez es más difícil desarrollar productos y servicios diferentes. Esta saturación también se da en la comunicación con la infoxicación a la que diariamente somos sometidos por parte de empresas, organizaciones e instituciones (Gómez-Nieto, 2016). Las marcas se han convertido en un elemento clave para aportar valor al consumidor más allá del producto y, para ello, necesitan establecer una relación con sus públicos (Prodigioso Volcán, 2019).

La sociedad de la información ha tenido como resultado que el consumidor y el público, en general, sea mucho más exigente (AEACP, 2017). Así, ha pasado de ser un agente pasivo en el proceso de comunicación, a un agente activo que no solo escucha, sino que además demanda y reivindica gracias al poder que las redes sociales le han otorgado. Por lo que, en un contexto globalizado donde lo que sucede a una marca en un mercado se replica rápidamente a otro es fundamental actuar con rapidez y saber responder adecuadamente (Llopis, 2015).

Ya no basta con tener un buen producto o servicio y tampoco comunicar sus características al consumidor, las empresas requieren contar con profesionales que sean capaces de aplicar estrategias y tácticas para gestionar adecuadamente la comunicación con sus pú- 
blicos a través de diferentes medios y soportes -especialmente el digital-, además de construir marcas que aporten valor al consumidor. Necesitan que sus profesionales desarrollen piezas creativas que permitan a un producto, una marca o una empresa conectar con su audiencia y sobresalir en este contexto de saturación publicitaria (Kaufmann, 2017; Álvarez-Flores et al., 2018).

La viabilidad de esta titulación a se ve reflejada por la tradición en la implantación en varias universidades españolas. En el curso 2019-2020, 35 centros contaban con un plan de estudios en Publicidad activo, de ellas, solo tres ofrecían estudios online, lo que mostraba la oportunidad de crecimiento en esta modalidad de enseñanza.

El diseño del plan de estudios del Grado en Publicidad y Relaciones Públicas en España se ha basado en un libro blanco elaborado por la Agencia Nacional de Evaluación de la Calidad y Acreditación (ANECA). Así, la agencia nacional dispone de documentos y herramientas para el programa VERIFICA, el cuál evalúa las propuestas de los planes de estudio de títulos diseñados en consonancia con el Espacio Europeo de Educación Superior. Entre ellos se encuentra la Guía de apoyo para la elaboración de la memoria para la solicitud de verificación de títulos oficiales y Libros Blancos para identificar las destrezas profesionales sobre las que vertebrar los planes de estudio. El Libro Blanco Títulos de Grado en Comunicación contiene la información necesaria para la confección de este título, aunque los principales responsables encargados de esta tarea en distintas universidades han destacado la escasez de producción científica centrada en los estudios de Publicidad y Relaciones Públicas (López et al., 2016), en el que también destaca el Libro Blanco para el diseño de titulaciones en la Economía Digital (Agenda Digital para España, 2015).

\section{COMPETENCIAS DEL ESTUDIANTE DE PUBLICIDAD}

Las competencias son el conjunto de conocimientos, habilidades, actitudes que se adquieren o desarrollan mediante experiencias formativas coordinadas, las cuales tienen el propósito de lograr conocimientos funcionales que den respuesta de modo eficiente a una tarea o problema de la vida cotidiana y profesional que requiera un proceso de enseñanza y aprendizaje (ANECA, 2015, p.21).

Así, entre las competencias clave del estudiante de Publicidad y que suponen un desafío materializarse a través de metodologías online son el trabajo en equipo, las habilidades de comunicación y la capacidad de conceptualización de ideas junto a la redacción de las mismas (Agüero et al., 2019 y Álvarez-Flores et al., 2018). De modo que, en esta memoria del Grado en Publicidad se incluyen como competencias fundamentales:

1. Habilidad para exponer en público trabajos publicitarios ante una audiencia especializada

2. Capacidad para trabajar en equipo en el entorno publicitario

3. Capacidad de generar nuevas ideas en el entorno publicitario

\section{LA LLEGADA DE LA PANDEMIA}

La memoria de verificación del Grado Online en Publicidad objeto de este estudio de caso fue aprobada por ANECA el 13 de enero de 2020 para su implementación en el curso 2020-21. De modo que, el proceso previo de preparación, así como el arranque del curso estuvo marcado por la pandemia sanitaria.

El sábado 14 de marzo de 2020 el Gobierno de España en respuesta al número creciente de casos de COVID-19 declaró el estado de alarma, lo que conllevó como principal medida la imposición de una cuarentena nacional para hacer frente a esta pandemia y, en consecuencia, un cambio de hábitos por parte de los consumidores. Los españoles vivieron catorce semanas de estado de alarma, durante el cual hubo un confinamiento domiciliario nacional (14 de marzo-2 de mayo) y un periodo de desescalada con un progresivo levantamiento de restricciones por territorios (4 de mayo-21 de junio). 
El confinamiento supuso la suspensión de las clases presenciales y la necesidad de pasar a online, con el consiguiente riesgo de distracción de los estudiantes. De hecho, el confinamiento afectó al consumo de medios con un incremento de los mismos (Gil et al., 2020).

Por otro lado, a pesar de que estamos ante una universidad adaptada al entorno online, la nueva situación provocada por la necesidad de quedarse en casa y teletrabajar supuso un notable incremento de la asistencia a clase por parte de los estudiantes ${ }^{1}$. Además, las propias recomendaciones sanitarias supusieron la rápida puesta en marcha de un plan de acción que conllevó que los exámenes, que hasta el momento eran presenciales, se hicieran de forma online ${ }^{2}$.

\section{OBJETIVOS Y METODOLOGÍA}

El objetivo de esta investigación es registrar la experiencia de poner en marcha un grado online de nueva impartición en un contexto excepcional de emergencia sanitaria. Se trata de compartir las prácticas diseñadas desde dirección académica con el propósito de crear un espacio de aprendizaje virtual que fomente y facilite el aprendizaje, el intercambio y el trabajo en equipo en un momento en el que la vida profesional y personal tanto de docentes como del alumnado se vio alterada. El valor añadido de esta investigación es que se describen dinámicas académicas en una modalidad docente ya de partida no presencial y que igualmente se vio afectada por esta situación de excepción. En concreto, se detallan las prácticas llevadas a cabo en el proceso de elaboración de contenidos, en las reuniones con el claustro del $1^{\circ}$ cuatrimestre del curso 2020-2021, en el enfoque de las sesiones en directo, en la preparación de encuentros con los alumnos y en el diseño de exámenes.

Por lo anterior, esta investigación tiene un carácter exploratorio y pretende compartir los resultados de una experiencia de coordinación académica

1 Véase: https://cincodias.elpais.com/cincodias/2020/08/04/extras/1596554008_919488.html

2 Véase: https://www.unir.net/actualidad/vida-academica/unir-cancela-toda-la-actividad-presencial-y-adopta-medidas-excepcionales-para-combatir-el-coronavirus/ que pueda aportar a otros colegas, no para probar una teoría. Así se prefirió una metodología cualitativa para primar la reflexión sobre la estrategia de enseñanza y aprendizaje en el aula.

Como técnica se empleó la observación participante: uno de los investigadores ejerció de "director académico-observador", funcionando como instrumento de recogida de datos. De esta forma el investigador forma parte del escenario de estudio, se involucra e interacciona con los informantes registrando la información de modo natural y no intrusivo (Taylor y Bogdan, 1986). Para compilar la información en este proceso de observación, el coordinador recopiló a través de notas las reflexiones y decisiones tomadas después de cada reunión y encuentro con los agentes implicados (claustro y alumnos), con el objetivo de tener un registro día a día y poder consultarlo como material para la posterior divulgación de esta experiencia académica.

\section{IMPLANTACIÓN DEL GRADO EN CONTEXTO COVID-19}

El proceso previo de la puesta en marcha. Elaboración de contenidos

El objetivo de la titulación, tal y como figura en el Suplemento Europeo al Título del estudiante, es capacitar al alumno con los conocimientos y habilidades necesarias para desempeñar la labor publicitaria y comunicativa en un entorno cambiante y marcadamente digital. El alumno será capaz de ocupar puestos creativos, emprender metodologías de investigación que permitan conocer al consumidor, planificar campañas y acciones en medios, así como gestionar adecuadamente la comunicación de una marca y sus públicos. Todo ello bajo un contexto de excepcionalidad en el que la motivación, la cercanía, la personalización y el acompañamiento resultaban más fundamentales que nunca, lo que supone todo un reto para un grado que empieza a escribir su historia.

Para conseguirlo, el principal reto se encuentra en aplicar la metodología online propia de esta universidad, pero en un contexto marcado por la pandemia. Primero, porque necesitábamos dejar claro nuestro ele- 
mento diferencial teniendo en cuenta las adversidades a las que se enfrentaban aquellas titulaciones que se habían visto de forma forzosa a adaptarse al entorno online y en la que esta institución ya tenía solvencia. En segundo lugar, porque suponía una oportunidad para aquellos estudiantes más jóvenes que comenzarían sus estudios universitarios en 2020-2021 y que elegirían, con motivo de la crisis sanitaria, cursar su educación superior en línea. Finalmente, porque pocos centros ofrecían esta titulación online, de modo que suponía un desafío adquirir las competencias del título a través de metodología virtual.

Así, el perfil de alumno del grado fue precisamente el esperado. Según fuentes de la propia universidad, un $44 \%$ tiene entre 17 y 24 años, un $20 \%$ entre 25 y 29 años, un $18 \%$ entre 30 y 34 años, un $4 \%$ entre 35 y 39 años y el $13 \%$ más de 40 años, lo que hace una edad media de 27,3 para este título, cuando lo común en un alumno de Grado en esta universidad es de 33 años $^{3}$. De modo que, el primer paso fue preparar los materiales pedagógicos, a disposición de los estudiantes en el campus virtual y que consta de: ideas clave, píldoras audiovisuales, recursos complementarios, test de autoaprendizaje.

El curso académico 2020-2021 arrancó con el primer año de la titulación. Durante esta primera etapa se contemplan aquellas asignaturas básicas prevenientes de las Ciencias Sociales y Jurídicas, así como Artes y Humanidades como: Lengua Española, Habilidades de Comunicación Oral, Historia del Mundo Actual, Tecnologías Multimedia y Sociedad o Teoría de la Comunicación, entre otras. Como materias específicas de la Publicidad destacan en este primer año dos, Redacción Publicitaria en el primer cuatrimestre y La Agencia de Publicidad en el segundo cuatrimestre.

En el diseño del material creado para estas asignaturas se puso el foco en la generación de conocimientos enmarcados en el entorno virtual, con el objetivo de llevar a cabo prácticas que contribuyesen a sensibilizar a los alumnos en el uso y aprovechamiento de las tecnologías digitales, a mostrar experiencias de eva-

3 Véase: https://www.unir.net/universidad-online/nuestros-alumnos/ https://www.libremercado.com/2020-11-07/caso-de-unir-proeduca-coronavirus-catapulta-exito-ensenanza-online-6671733/ luación de los conocimientos adquiridos y a proponer modelos colaborativos entre estudiantes.

Arranque del curso 2020-2021: buenas prácticas

Reuniones con el Claustro

El arranque del curso 2020-2021 comenzó con la reunión general entre el profesorado y la dirección del título con el propósito de proporcionar indicaciones para enriquecer la impartición de las sesiones en directo, principal vía de la que dispone el docente para conectar de forma síncrona con sus estudiantes. De todos los recursos es el más idóneo para trabajar las competencias clave del título: la exposición en público, el trabajo en equipo y la generación de nuevas ideas. Consecuentemente se hace un refuerzo especial en la formación para ofrecer unas sesiones que fomenten la asistencia en directo, la participación y un contacto más personal y cercano con el alumno, especialmente en una situación de confinamiento en la que el estado anímico influye mucho más en la actitud y motivación. Igualmente, también se hizo hincapié en el perfil más joven de estudiante matriculado en este grado, para tenerlo en cuenta en la búsqueda de ejemplos y de insights.

Tras la celebración del claustro general, se trabajó de forma específica con cada profesor a través de Microsoft Teams y/o Adobe Connect con el objetivo de adaptar la materia publicitaria al entorno online, a las características de los nuevos estudiantes y a la situación de confinamiento vivida tanto por profesores como por alumnos.

El objetivo de estas sesiones específicas de trabajo con cada profesor responsable de las asignaturas del primer cuatrimestre del curso 2020-2021 ha sido proporcionar información que ayudase a los docentes a preparar y enfocar cada asignatura en concreto a las necesidades de la titulación en el marco de la innovación docente propio de la facultad a la que está adscrita, así como a las circunstancias coyunturales marcadas por la pandemia. Así, se resaltaron los siguientes hitos docentes a conseguir en cada asignatura del primer cuatrimestre: 
1. Habilidades de Comunicación Oral. Compartir ejemplos orientados a Publicidad en las clases presenciales virtuales; necesidad de que los estudiantes practiquen competencias orales conectando micro y/o cámara siempre que sea posible. El examen debe fomentar la práctica, incorporar para ello casos.

2. Historial del Mundo Actual. Compartir ejemplos orientados a Publicidad en clases presenciales virtuales siempre que sea posible, haciendo la asignatura más atractiva con recursos audiovisuales. Incorporar una pregunta práctica de Publicidad en el examen.

3. Técnicas de Búsqueda y Uso de la Información. Es una asignatura muy orientada a periodismo, se requiere ejemplificar en Publicidad. Compartir pantalla en clases. Examen práctico.

4. Lengua Española. Completar las clases presenciales virtuales con casos prácticos en los foros. Examen práctico.

5. Redacción Publicitaria. Se trata de la primera asignatura de la disciplina que aparece en el plan de estudios. Se enfocó como una materia muy práctica en el que es fundamental que el estudiante "aprenda haciendo". Por tanto, resultó clave el diseño y la puesta en marcha de dinámicas de competencia oral y escrita para afianzar los conceptos teóricos tanto en las clases en directo como a través de los foros. Con este tipo de ejercicios complementarios se buscaba impulsar la asistencia a clase en directo y la participación del estudiante tanto a través de micro como de cámara. A modo de ejemplo:

» Para que el estudiante practicase como a través del lenguaje escrito se conceptualiza el pensamiento abstracto, se propuso el ejercicio de poner un título a determinados cuadros abstractos.

» A partir de productos sugerentes y atípicos, se instó a crear textos persuasivos.
» Una de las bases de la creatividad es la combinación de mundos aparentemente desconectados, por ello se diseñaron varios ejercicios de este estilo en los que se les obligaba a superar los bloques creativos: ¿Seríamos capaces de realizar una declaración de amor empleando nuestra jerga publicitaria? Prohibido el uso de palabras típicas: amor, emoción, pasión, querer, deseo, cariño, afecto...

»En los temas centrales de la asignatura, como son los relacionados con el proceso de conceptualización de la idea creativa, se les animó a que redefiniese y actualizasen el concepto de amor en el siglo XXI.

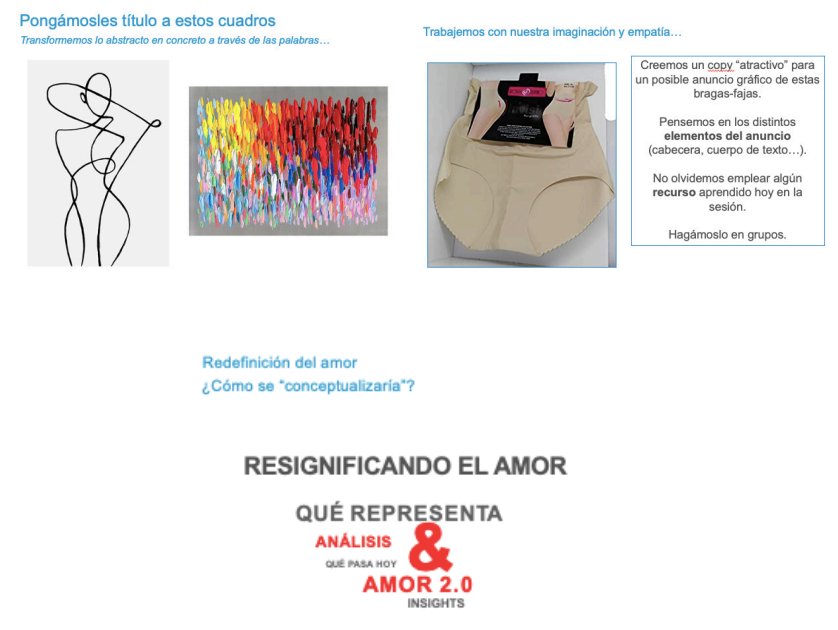

Figura 1. Ejemplo de actividades complementarias en Redacción Publicitaria

Además, bajo el propósito de fomentar la rutina de escribir de forma frecuente, se organizó un concurso de redacción de participación voluntaria y en equipos. Se propuso que los grupos de trabajo asumiesen el rol de community manager de una marca y que publicasen para dicha marca semanalmente una entrada en Instagram sobre un tema de actualidad planteado por el docente. El concurso se desarrolló a través del 
foro del aula creando uno en específico para esta dinámica. Se buscó que el alumno tomase el hábito de redactar de forma periódica combinando la creatividad y el humor, además de fomentar el trabajo en equipo y el contacto entre alumnos más allá de lo estrictamente obligatorio, creando un ambiente enriquecedor y dinámico característico del mundo universitario.

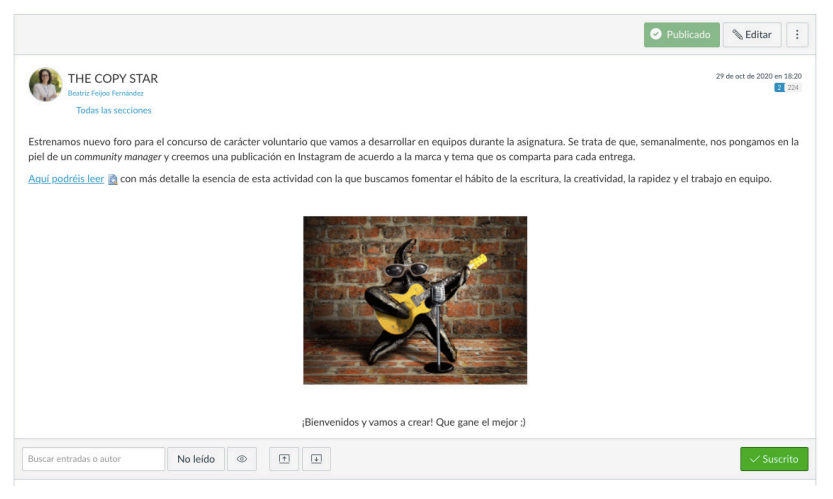

Figura 2. Ejemplo para dinamizar el uso del foro

Durante todo el cuatrimestre se hizo un seguimiento por parte de coordinación académica con el objetivo de velar por el cumplimiento de los resultados de aprendizaje esperados de la metodología empleada en cada materia.

\section{Las sesiones en directo}

Desde coordinación académica del título se destacó desde el primer momento la importancia de aprovechar las sesiones en directo para mantener un contacto más cercano con el estudiante, especialmente en estos momentos: aportó valor conectarse al aula virtual con tiempo de antelación para disponer de unos minutos previos para interactuar con los alumnos de una manera más informal y crear un ambiente de intercambio más allá de lo estrictamente académico.

Igualmente, se intentaron diseñar clases más participativas mediante recursos prácticos como preguntas, retos, planteamiento de problemas mediante encuestas de respuesta rápida y trabajos en equipos, dos dinámicas posibles gracias a la herramienta de Adobe Connect.
Igualmente, al finalizar la clase y su grabación, también se intentaba recuperar un momento de distensión para cerrar la sesión con ánimo y actitud positiva ante las circunstancias excepcionales que se estaban viviendo.

Puntos de encuentro con los alumnos más allá de las sesiones en directo obligatorias

Con el objetivo de mantener el interés de los estudiantes, fomentar el pensamiento crítico y contribuir a su formación complementaria, se llevaron a cabo una serie de masterclass en las que participaron la coordinadora académica y profesores del primer curso del título. Este tipo de sesiones extraacadémicas ha sido una muestra del trabajo en equipo entre el claustro.

Las masterclass llevadas a cabo en este primer cuatrimestre se realizaron a través de Adobe Connect y se ofrecieron de forma transversal a todos los títulos de la facultad que está adscrito el Grado en Publicidad, sirviendo así de punto de encuentro entre estudiantes de diversas titulaciones. Las clases quedaron grabadas para su visionado en diferido por parte de aquellos estudiantes que no pudieran acceder en directo. Asimismo, se hizo un resumen del aprendizaje a través de plataformas de divulgación de la universidad:

Las sesiones del primer cuatrimestre del curso 2020-2021 fueron:

» Aptitudes y habilidades para trabajar en publicidad (22 de octubre de 2020). El sector publicitario es especialmente cambiante. Se necesita una reflexión continuada sobre las habilidades, las competencias y los perfiles que exige la industria. Se demandan profesionales cada vez más híbridos y transversales que tengan aptitudes multidisciplinares. Trabajo en equipo, proactividad, capacidad analítica, dotes para la comunicación, empatía ... La publicidad se ha vinculado además siempre a la creatividad, pero ¿sigue siendo la creatividad una característica fundamental? ¿Cuáles son realmente las cualidades que deben tener un publicista? 
El anuncio de la lotería siempre nos acompaña: la cita anual para compartir. (26 de noviembre de 2020) ${ }^{4}$. Según se acercan las fechas navideñas, tanto los amantes de la Publicidad como los que no, esperan con ansia el anuncio de la Lotería. Este año más que nunca- como propone la campaña de la Lotería $\mathrm{Na}$ cional-, compartamos como siempre, compartamos como nunca. Y como es el sorteo que nos une, una sesión compartir nuestras impresiones sobre el tan conocido anuncio de Lotería Navidad 2020.

Masterclass la función social de la publicidad. Comunicando en tiempos del COVID-19 (19 de enero de 2020)5. Los gobiernos tienen constantemente la necesidad de cambiar percepciones o intentar cambiar hábitos para mejorar la convivencia, evitar problemas sanitarios, o de seguridad. Cada año las administraciones públicas desarrollan campañas institucionales contra la violencia de género, el buen comportamiento al volante, el reciclaje, ... pero en 2020, como todos, se han enfrentado a un reto verdaderamente difícil, educar a los ciudadanos en un tiempo récord y de forma sostenida a seguir comportamientos totalmente nuevos que atañen a la totalidad de su forma de vivir.

Por otro lado, en esta modalidad virtual no se puede olvidar la utilidad y versatilidad de los foros habilitados en las aulas virtuales de cada asignatura. A través de ellos se busca predisponer al alumno al contenido de las propias clases e invitarles a la reflexión y el autoaprendizaje (despertar curiosidades). Si bien por defecto, el aula virtual se abre con un foro de dudas, desde coordinación se fomentó la apertura de más espacios a través de los cuales "mantener vivos" los te-

4 Véase: https://www.unir.net/marketing-comunicacion/revista/el-anuncio-de-la-loteria-siempre-nos-acompana-la-cita-anual-para-compartir/

5 Véase: https://www.unir.net/marketing-comunicacion/revista/publicidad-institucional-comunicar-en-tiempos-de-covid-19/ mas que más interés despierten de las sesiones en directo. Se trata, en definitiva, de que los foros alimenten la atención del alumno entre clase y clase.

\section{Resultados obtenidos}

Terminado el primer cuatrimestre del curso $2020-2021$, se puede afirmar que los alumnos realizaron al examen en convocatoria ordinaria habiendo puesto en práctica los conocimientos teóricos de la materia y en consecuencia con puntos de evaluación continua. Comprenden la necesidad de investigar y conocer muy bien el producto y empresa para la que van a realizar la campaña, así como el público al que se dirigen. Y la práctica oral la disfrutan. Pero incluso aquellos alumnos que no se animaron a realiza la exposición oral de sus trabajos sí asistieron a las sesiones para conocer cómo sus compañeros han dado respuesta de manera creativa al problema del anunciante y participan en esta exposición de manera activa.

Sirvan como ejemplo las calificaciones del primer cuatrimestre en convocatoria ordinaria. De media se consiguió una tasa de aprobados del $84 \%$, un $90 \%$ de tasa de éxito (comparativa entre los aprobados y presentados) y una satisfacción entre el alumnado de $8,7 / 10$.

\section{CONCLUSIONES}

El valor añadido de esta investigación es compartir las experiencias docentes de una universidad de modalidad virtual que busca la formación integral del alumno. En este caso se tuvo en cuenta además la variable de excepcionalidad introducida por la pandemia sanitaria del coronavirus que ha causado cambios en la estrategia docente a corto plazo del sistema universitario español y mundial.

Si bien en este caso ya se parte de una docencia virtual y que fue el cambio más notorio que experimentó la mayoría de las universidades del panorama español por causa de esta situación de emergencia, ello no significa que en las universidades originalmente online no tuvieran que adaptar su enseñanza a la nueva situación. 
La misión de ofrecer una educación personalizada y el reto de romper con la premisa de impersonalidad y frialdad asociada con la educación a distancia marcaron especialmente la puesta en marcha de este Grado Online en Publicidad en un momento histórico extremo en el que el fundamento de la persona adquiere todavía más valor, sin perder de vista la consecución de las competencias clave de esta disciplina: habilidad para exponer en público; capacidad para generar nuevas ideas y para trabajar en equipo en el entorno publicitario.
De ahí que las propuestas descritas (reuniones personalizadas entre profesores y coordinación; dinamización foros; retos en clases en directo, formación complementaria...) estuviesen destinadas a fomentar un espacio de encuentro, de debate y de enriquecimiento entre los distintos agentes implicados (coordinación, claustro, alumnos y tutores) con el objetivo de construir una comunidad en un momento de extraordinaria necesidad vital.

\section{REFERENCIAS}

» Agencia Nacional de Evaluación de la Calidad y Acreditación [ANECA] (2015): Guía de apoyo para la elaboración de la memoria de verificación de títulos oficiales universitarios. (Grado y Máster. Última actualización 01-12-2015). Recuperado de: http:// www.aneca.es/content/download/12155/136031/file/verifica_ gm_guia_V05.pdf

» Agencia Nacional de Evaluación de la Calidad y Acreditación [ANECA] (2005). Libro Blanco Títulos de Grado en Comunicación. Recuperado de: http://www.aneca.es/var/media/150336/libroblanco_comunicacion_def.pdf

» Agenda Digital para España (2015). Libro Blanco para el diseño de titulaciones en la Economía Digital. Recuperado de: http:// www.aneca.es/content/download/13246/164004/file/Libro\%20 Blanco\%20para\%20el\%20dise\%C3\%B10\%20de\%20las\%20titulaciones $\% 20$ universitarias $\% 20$ en $\% 20$ el $\% 20$ marco $\% 20 \mathrm{de} \% 20$ la\%20Econom\%C3\%ADa\%20Digital.pdf

» Agüero Pérez, M.M.; López Alonso, E.; López Fraile, L.A. y Moreno López, B. (2019): Implicación de los stakeholders en la creación del Grado en Publicidad en la Universidad Europea. Imbricación del sector profesional en el entorno académico. Revista Latina de Comunicación Social, 74, 50 a 72. https://doi.org/10.4185/RLCS2019-1321

» Álvarez-Flores, Erika P.; Núñez-Gómez, Patricia; Olivares-Santamarina, José P. (2018). Perfiles profesionales y salidas laborales para graduados en Publicidad y Relaciones públicas: de la especialización a la hibridación. El profesional de la información, 27 (1), 136-147. https://doi.org/10.3145/epi.2018.ene.13

» Asociación Española de Agencias de Comunicación Publicitaria (AEACP) (2017). "Prólogo de la AEACP". En: Kaufmann, J. La transformación digital de la agencia de publicidad. Estudio empírico del caso español. pp.5-9. Universidad de Navarra. Recuperado de: https://creatividadtransformadora.es/wp-content/ uploads/2017/06/Transformaci\%C3\%B3n_digital_alta_interact-2.pdf

» Castellanos Sánchez, A., y Martínez De la Muela, A. (2013). "Trabajo en equipo con Google Drive en la universidad online”. Innovación educativa (México, DF), 13(63), pp. 75-94. http://www.scielo.org. mx/scielo.php?script=sci_arttext\&pi$\mathrm{d}=\mathrm{S} 1665-26732013000300006 \& \mid \mathrm{ng}=$ es\&tlng=
» Gil-Torres, A., Martín-Quevedo, J., Gómez-García, S. \& San José-De la Rosa, C. (2020). El coronavirus en el ecosistema de los dispositivos móviles: creadores, discursos y recepción. Revista Latina de Comunicación Social, 78, 329-358. https://www.doi.org/10.4185/ RLCS-2020-1480

» Gómez Nieto, B. (2016). El consumidor ante la infoxicación en el discurso periodístico. Estudios sobre el Mensaje Periodístico, 22 (1), 313-327. https://doi.org/10.5209/rev_ESMP.2016.v22. n1.52598

» Kaufmann, J. (2017). La transformación digital de la agencia de publicidad. Estudio empírico del caso español. Universidad de Navarra. Recuperado de: https://creatividadtransformadora.es/ wp-content/uploads/2017/06/Transformaci\%C3\%B3n_digital_ alta_interact-2.pdf

» Llopis, E. (2015). Crear la marca global. ESIC Editorial.

» López, A. y Nagel, J. (2018), “Qué es una educación de calidad? La docencia en el siglo XXI". En J. A. Guzmán, J. I. Brito e I. Illanes (Eds.). La universidad en debate: 18 miradas sobre una controversia. Universidad de los Andes, Santiago de Chile, Chile (pp. 171-184).

» López, S.; Papí, N. y Martín, M. (2016). La revisión de los grados universitarios: valoración de la convergencia europea del Grado en Publicidad y Relaciones Públicas en España. doxa.comunicación, 23, 47-71. https://doi.org/10.31921/doxacom.n23a2

» Moreno Castañeda, M., Chan Núñez, M. E., Flores Briseño, M. M, Hernández Figueroa V. G., Córdova Soltero, H.J, y Coronado Ramírez, G. (2010). Modelo Educativo del Prodigioso Volcán (2019). Cómo construir marcas para el siglo XXI. Recuperado de: https:// www.prodigiosovolcan.com/sismogramas/como-crear-marcas/

» Sistema de Universidad Virtual. Universidad de Guadalajara, Guadalajara, México. http://148.202.167.116:8080/jspui/bitstream/123456789/1829/3/Modelo\%20Educativo\%20SUV.pdf

» Sangrà, A. (2001). La calidad en las experiencias virtuales de educación superior. http://e-spacio.uned.es/fez/eserv/bibliuned:1065/n04sangra01.pdf

» Taylor, S.J., y Bogdan, R. (1986), Introducción a los métodos cualitativos de investigación. Paidós, Barcelona.

» Tourón, J., y Martín Rodríguez, D. (2019). Aprender y enseñar en la universidad hoy. Una guía práctica para profesores. Universidad Internacional de la Rioja, La Rioja, España. 